\title{
Determination of RNA hydroxylmethylation in mammals by mass spectrometry analysis
}

Xue-Jiao You,,${ }^{1,2} \ddagger$ Ting Liu,,${ }^{1} \ddagger$ Cheng-Jie Ma, ${ }^{1}$ Chu-Bo Qi,${ }^{1,3}$ Yongjia Tong,${ }^{4}$ Xiaolu Zhao, ${ }^{4}$ Bi-Feng Yuan, ${ }^{1,2, *}$ Yu-Qi Feng ${ }^{1}$

${ }^{1}$ Key Laboratory of Analytical Chemistry for Biology and Medicine (Ministry of Education), Department of Chemistry, Wuhan University, Wuhan 430072, P.R. China.

${ }^{2}$ Sauvage Center for Molecular Sciences, Wuhan University, Wuhan 430072, P.R. China.

${ }^{3}$ Hubei Cancer Hospital, Tongji Medical College, Huazhong University of Science and Technology, Wuhan 430079, P.R. China.

${ }^{4}$ Hubei Key Laboratory of Cell Homeostasis, College of Life Sciences, Wuhan University, Wuhan 430072, P.R. China.

t These authors contributed equally to this work.

* Corresponding author: Bi-Feng Yuan. Tel: +86-27-68755595; Fax: +86-27-68755595;

E-mail: bfyuan@whu.edu.cn 
The Supporting Information includes following items:

Page S3 Table S1. The information of nucleoside and modified nucleoside standards.

Page S4 Table S2. The MRM transitions and optimal parameters for the analysis of nucleosides by mass spectrometry.

Page S5 Table S3. Linearities, calibration curves, LODs and LOQs for the analysis of modified nucleosides by LC-ESI-MS/MS.

Page S6 Table S4. The accuracy and precision for the determination of $\mathrm{m}^{6} \mathrm{~A}$.

Page 7 Table S5. The accuracy and precision for the determination of $\mathrm{m}^{5} \mathrm{C}$.

Page S8 Table S6. The accuracy and precision for the determination of $\mathrm{m}^{2} \mathrm{G}$.

Page S9 Table S7. The accuracy and precision for the determination of $\mathrm{hm}^{6} \mathrm{~A}$.

Page S10 Table S8. The accuracy and precision for the determination of $\mathrm{hm}^{5} \mathrm{C}$.

Page S11 Table S9. The accuracy and precision for the determination of $\mathrm{hm}^{2} \mathrm{G}$.

Page S12 Figure S1. The chemical reactions for the synthesis of (A) $\mathrm{hm}^{6} \mathrm{~A}$ and (B) $\mathrm{hm}^{2} \mathrm{G}$.

Page S13-S14 Figure S2. Characterization of the synthesized $\mathrm{hm}^{6} \mathrm{~A}$ and $\mathrm{hm}^{2} \mathrm{G}$ by NMR. (A) hm ${ }^{6} \mathrm{~A}$. (B) hm ${ }^{2} \mathrm{G}$.

Page S15 Figure S3. Evaluation of the stability of $\mathrm{hm}^{6} \mathrm{~A}$ and $\mathrm{hm}^{2} \mathrm{G}$ in neutral conditions at $25^{\circ} \mathrm{C}$.

Page S16 Figure S4. Evaluation of the signal intensities of normal nucleosides in neutral buffer and traditional enzymatic digestion buffer.

Page S17 FigureS5. Confirmation of the detected $h m^{6} \mathrm{~A}, \mathrm{hm}^{5} \mathrm{C}$, and $\mathrm{hm}^{2} \mathrm{G}$ by spiking the standards to the tested samples.

Page S18 Figure S6. The extracted-ion chromatograms of the samples with only adding phosphodiesterase I or calf intestinal alkaline phosphatase (CIAP) and omitting the RNA sample.

Page S19 Figure S7. The typical extracted-ion chromatograms and product ion spectra of $\mathrm{m}^{6} \mathrm{~A}, \mathrm{~m}^{5} \mathrm{C}$, and $\mathrm{m}^{2} \mathrm{G}$ from standards and from RNA of HEK293T cells.

Page S20 Figure S8. Confirmation of the detected $\mathrm{m}^{6} \mathrm{~A}, \mathrm{~m}^{5} \mathrm{C}$, and $\mathrm{m}^{2} \mathrm{G}$ by spiking the standards to tested samples. 
Table S1. The information of nucleoside and modified nucleoside standards.

\begin{tabular}{lccccc}
\hline Nucleosides & $\begin{array}{c}\text { Abbrev } \\
\text { iation }\end{array}$ & $\begin{array}{c}\mathrm{CAS} \\
\text { number }\end{array}$ & $\begin{array}{c}\text { Molecular } \\
\text { formula }\end{array}$ & $\begin{array}{c}\text { Molecular } \\
\text { weigh }\end{array}$ & Company \\
\hline cytidine & $\mathrm{C}$ & $65-46-3$ & $\mathrm{C}_{9} \mathrm{H}_{13} \mathrm{~N}_{3} \mathrm{O}_{5}$ & 243.22 & Sigma-Aldrich \\
uridine & $\mathrm{U}$ & $58-96-8$ & $\mathrm{C}_{9} \mathrm{H}_{12} \mathrm{~N}_{2} \mathrm{O}_{6}$ & 244.20 & Sigma-Aldrich \\
guanosine & $\mathrm{G}$ & $118-00-3$ & $\mathrm{C}_{10} \mathrm{H}_{13} \mathrm{~N}_{5} \mathrm{O}_{5}$ & 283.24 & Sigma-Aldrich \\
adenosine & $\mathrm{A}$ & $58-61-7$ & $\mathrm{C}_{10} \mathrm{H}_{13} \mathrm{~N}_{5} \mathrm{O}_{4}$ & 267.24 & Sigma-Aldrich \\
5-methylcytidine & $\mathrm{m}^{5} \mathrm{C}$ & $2140-61-6$ & $\mathrm{C}_{10} \mathrm{H}_{15} \mathrm{~N}_{3} \mathrm{O}_{5}$ & 257.24 & Sigma-Aldrich \\
$N^{2}$-methylguanosine & $\mathrm{m}^{2} \mathrm{G}$ & $2140-77-4$ & $\mathrm{C}_{11} \mathrm{H}_{15} \mathrm{~N}_{5} \mathrm{O}_{5}$ & 297.27 & Carbosynth \\
$N^{6}$-methyladenosine & $\mathrm{m}^{6} \mathrm{~A}$ & $1867-73-8$ & $\mathrm{C}_{11} \mathrm{H}_{15} \mathrm{~N}_{5} \mathrm{O}_{4}$ & 281.27 & Hanhong Chemical \\
5-hydroxymethylcytidine & $\mathrm{hm}^{5} \mathrm{C}$ & $19235-17-7$ & $\mathrm{C}_{10} \mathrm{H}_{15} \mathrm{~N}_{3} \mathrm{O}_{6}$ & 273.24 & Berry\&Associates \\
$N^{2}$-hydroxymethylguanosine & $\mathrm{hm}^{2} \mathrm{G}$ & - & $\mathrm{C}_{11} \mathrm{H}_{15} \mathrm{~N}_{5} \mathrm{O}_{6}$ & 313.27 & Synthesized in the \\
$N^{6}$-hydroxymethyladenosine & $\mathrm{hm}^{6} \mathrm{~A}$ & - & $\mathrm{C}_{11} \mathrm{H}_{15} \mathrm{~N}_{5} \mathrm{O}_{5}$ & 297.27 & current study \\
\hline
\end{tabular}


Table S2. The MRM transitions and optimal parameters for the analysis of nucleosides by mass spectrometry.

\begin{tabular}{|c|c|c|c|c|c|c|c|c|}
\hline Nucleosides & Abbreviation & Q1 & Q3 & DP & EP & $\mathrm{CE}$ & CXP & RT (min) \\
\hline cytidine & $\mathrm{C}$ & 244.1 & 112.1 & 29.3 & 4.1 & 22.6 & 5.0 & 5.8 \\
\hline uridine & $\mathrm{U}$ & 245.1 & 113.0 & 18.4 & 9.2 & 21.7 & 3.0 & 7.2 \\
\hline guanosine & G & 284.1 & 152.1 & 25.0 & 4.2 & 24.8 & 4.6 & 13.5 \\
\hline adenosine & A & 268.1 & 136.1 & 33.0 & 6.0 & 25.7 & 3.0 & 18.1 \\
\hline 5-methylcytidine & $\mathrm{m}^{5} \mathrm{C}$ & 258.1 & 126.1 & 19.3 & 7.9 & 23.7 & 3.6 & 2.5 \\
\hline$N^{2}$-methylguanosine & $\mathrm{m}^{2} \mathrm{G}$ & 298.1 & 166.1 & 19.0 & 5.0 & 19.0 & 2.0 & 17.3 \\
\hline$N^{6}$-methyladenosine & $\mathrm{m}^{6} \mathrm{~A}$ & 282.1 & 150.1 & 41.5 & 6.2 & 30.2 & 2.4 & 22.1 \\
\hline 5-hydroxymethylcytidine & $\mathrm{hm}^{5} \mathrm{C}$ & 274.1 & $142.1 / 124.1$ & 30.0 & 3.0 & 16.0 & 4.0 & 6.0 \\
\hline$N^{2}$-hydroxymethylguanosine & $\mathrm{hm}^{2} \mathrm{G}$ & 314.1 & 164.1 & 33.0 & 5.0 & 30.0 & 5.0 & 14.5 \\
\hline$N^{6}$-hydroxymethyladenosine & $\mathrm{hm}^{6} \mathrm{~A}$ & 298.1 & 136.1 & 25.0 & 5.0 & 26.0 & 3.0 & 18.9 \\
\hline
\end{tabular}

Q1: the first quadrupole; Q3: the third quadrupole; RT: retention time; DP: declustering potential; EP: entrance potential; CE: collision energy; CXP: collision cell exit potential. 
Table S3. Linearities, calibration curves, LODs and LOQs for the analysis of modified nucleosides by LC-ESI-MS/MS.

\begin{tabular}{|c|c|c|c|c|c|c|}
\hline \multirow{2}{*}{$\begin{array}{c}\text { Molar } \\
\text { ratios (\%) }\end{array}$} & \multirow{2}{*}{$\begin{array}{c}\text { Linear range } \\
(\%)\end{array}$} & \multicolumn{3}{|c|}{ Calibration curve data } & \multirow{2}{*}{ LOD (fmol) } & \multirow{2}{*}{ LOQ (fmol) } \\
\hline & & Slope & Intercept & $\mathrm{R}^{2}$ & & \\
\hline $\mathrm{m}^{5} \mathrm{C} / \mathrm{C}$ & $0.02-5$ & $3.16 \times 10^{-1}$ & $1.03 \times 10^{-2}$ & 0.9989 & 0.6 & 2.2 \\
\hline $\mathrm{m}^{2} \mathrm{G} / \mathrm{G}$ & $0.02-5$ & $5.17 \times 10^{-2}$ & $3.40 \times 10^{-3}$ & 0.9908 & 0.7 & 2.4 \\
\hline $\mathrm{m}^{6} \mathrm{~A} / \mathrm{A}$ & $0.02-5$ & $2.01 \times 10^{-2}$ & $8.29 \times 10^{-5}$ & 0.9998 & 0.9 & 3.2 \\
\hline $\mathrm{hm}^{5} \mathrm{C} / \mathrm{C}$ & $0.00002-0.05$ & $9.06 \times 10^{-2}$ & $-4.07 \times 10^{-5}$ & 0.9998 & 1.0 & 3.5 \\
\hline $\mathrm{hm}^{2} \mathrm{G} / \mathrm{G}$ & $0.0002-0.05$ & $9.38 \times 10^{-3}$ & $-6.28 \times 10^{-7}$ & 0.9990 & 1.3 & 4.3 \\
\hline $\mathrm{hm}^{6} \mathrm{~A} / \mathrm{A}$ & $0.0002-0.05$ & $1.04 \times 10^{-2}$ & $7.85 \times 10^{-6}$ & 0.9984 & 1.6 & 5.1 \\
\hline
\end{tabular}


Table S4. The accuracy and precision for the determination of $\mathrm{m}^{6} \mathrm{~A}$.

\begin{tabular}{ccccc}
\hline & $\begin{array}{c}\text { Nominal } \\
\mathrm{m}^{6} \mathrm{~A} / \mathrm{A} \%\end{array}$ & 0.020 & 0.20 & 5.00 \\
\hline \multirow{3}{*}{$\begin{array}{c}\text { Measured mean } 1 \\
\mathrm{~m}^{6} \mathrm{~A} / \mathrm{A} \%\end{array}$} & 0.020 & 0.214 & 5.133 \\
& $\mathrm{RSD}(\%)$ & 12.6 & 9.6 & 1.3 \\
& $\mathrm{RE}(\%)$ & 1.4 & 6.9 & 2.7 \\
& Measured mean & 0.019 & 0.198 & 5.223 \\
Day 2 & $\mathrm{~m}^{6} \mathrm{~A} / \mathrm{A} \%$ & 6.4 & 5.6 \\
$n=3$ & $\mathrm{RSD}(\%)$ & -3.3 & -0.8 & 4.5 \\
& $\mathrm{RE}(\%)$ & 0.021 & 0.183 & 5.089 \\
& Measured mean & & & \\
Day 3 & $\mathrm{m}^{6} \mathrm{~A} / \mathrm{A} \%$ & 5.5 & 4.9 & 2.9 \\
$n=3$ & $\mathrm{RSD}(\%)$ & 4.9 & -8.4 & 1.8 \\
\hline
\end{tabular}


Table S5. The accuracy and precision for the determination of $\mathrm{m}^{5} \mathrm{C}$.

\begin{tabular}{|c|c|c|c|c|}
\hline & $\begin{array}{l}\text { Nominal } \\
\mathrm{m}^{5} \mathrm{C} / \mathrm{C} \%\end{array}$ & 0.020 & 0.20 & 5.00 \\
\hline Day 1 & $\begin{array}{c}\text { Measured mean } \\
\mathrm{m}^{5} \mathrm{C} / \mathrm{C} \%\end{array}$ & 0.018 & 0.205 & 5.146 \\
\hline \multirow[t]{2}{*}{$n=3$} & RSD (\%) & 5.4 & 5.3 & 1.3 \\
\hline & RE (\%) & -7.6 & 2.7 & 2.9 \\
\hline Day 2 & $\begin{array}{c}\text { Measured mean } \\
\mathrm{m}^{5} \mathrm{C} / \mathrm{C} \%\end{array}$ & 0.019 & 0.198 & 4.764 \\
\hline \multirow[t]{2}{*}{$n=3$} & RSD (\%) & 8.7 & 10.1 & 8.0 \\
\hline & RE (\%) & -2.7 & -1.1 & -4.7 \\
\hline Day 3 & $\begin{array}{c}\text { Measured mean } \\
\mathrm{m}^{5} \mathrm{C} / \mathrm{C} \%\end{array}$ & 0.020 & 0.186 & 5.177 \\
\hline \multirow[t]{2}{*}{$n=3$} & RSD (\%) & 7.1 & 6.6 & 2.5 \\
\hline & RE (\%) & -1.5 & -7.2 & 3.5 \\
\hline
\end{tabular}


Table S6. The accuracy and precision for the determination of $\mathrm{m}^{2} \mathrm{G}$.

\begin{tabular}{|c|c|c|c|c|}
\hline & $\begin{array}{l}\text { Nominal } \\
\mathrm{m}^{2} \mathrm{G} / \mathrm{G} \%\end{array}$ & 0.020 & 0.020 & 5.00 \\
\hline Day 1 & $\begin{array}{c}\text { Measured mean } \\
\mathrm{m}^{2} \mathrm{G} / \mathrm{G} \%\end{array}$ & 0.019 & 0.212 & 5.139 \\
\hline \multirow[t]{2}{*}{$n=3$} & RSD (\%) & 7.3 & 1.5 & 3.4 \\
\hline & $\mathrm{RE}(\%)$ & -3.7 & 6.1 & 2.8 \\
\hline Day 2 & $\begin{array}{l}\text { Measured mean } \\
\mathrm{m}^{2} \mathrm{G} / \mathrm{G} \%\end{array}$ & 0.020 & 0.193 & 4.876 \\
\hline \multirow[t]{2}{*}{$n=3$} & RSD (\%) & 4.6 & 4.0 & 0.8 \\
\hline & RE (\%) & 2.0 & -3.7 & -2.5 \\
\hline Day 3 & $\begin{array}{c}\text { Measured mean } \\
\mathrm{m}^{2} \mathrm{G} / \mathrm{G} \%\end{array}$ & 0.018 & 0.208 & 5.301 \\
\hline \multirow[t]{2}{*}{$n=3$} & RSD (\%) & 5.2 & 7.9 & 3.0 \\
\hline & RE (\%) & -8.5 & 4.2 & 6.0 \\
\hline
\end{tabular}


Table S7. The accuracy and precision for the determination of $\mathrm{hm}^{6} \mathrm{~A}$.

\begin{tabular}{|c|c|c|c|c|}
\hline & $\begin{array}{c}\text { Nominal } \\
\mathrm{hm}^{6} \mathrm{~A} / \mathrm{A} \%\end{array}$ & 0.00020 & 0.0020 & 0.050 \\
\hline Day 1 & $\begin{array}{c}\text { Measured mean } \\
\mathrm{hm}^{6} \mathrm{~A} / \mathrm{A} \%\end{array}$ & 0.00019 & 0.00210 & 0.05280 \\
\hline \multirow[t]{2}{*}{$n=3$} & RSD (\%) & 4.5 & 7.6 & 3.9 \\
\hline & RE (\%) & -5.1 & 4.9 & 5.6 \\
\hline \multirow{3}{*}{$\begin{array}{c}\text { Day } 2 \\
n=3\end{array}$} & $\begin{array}{c}\text { Measured mean } \\
\mathrm{hm}^{6} \mathrm{~A} / \mathrm{A} \%\end{array}$ & 0.00021 & 0.00175 & 0.05142 \\
\hline & RSD (\%) & 4.0 & 9.3 & 4.6 \\
\hline & RE $(\%)$ & 6.6 & -12.7 & 2.9 \\
\hline \multirow{3}{*}{$\begin{array}{c}\text { Day } 3 \\
n=3\end{array}$} & $\begin{array}{c}\text { Measured mean } \\
\mathrm{hm}^{6} \mathrm{~A} / \mathrm{A} \%\end{array}$ & 0.00019 & 0.00207 & 0.04803 \\
\hline & RSD (\%) & 6.9 & 9.4 & 2.1 \\
\hline & RE (\%) & -2.8 & 3.4 & -3.9 \\
\hline
\end{tabular}


Table S8. The accuracy and precision for the determination of $\mathrm{hm}^{5} \mathrm{C}$.

\begin{tabular}{ccccc}
\hline & Nominal & 0.000020 & 0.0010 & 0.050 \\
\hline \multirow{2}{*}{$\mathrm{hm}^{5} \mathrm{C} / \mathrm{C} \%$} & Measured mean & 0.000021 & 0.00110 & 0.05140 \\
Day 1 & $\mathrm{hm}^{5} \mathrm{C} / \mathrm{C} \%$ & 7.8 & 5.3 \\
$n=3$ & $\mathrm{RSD}(\%)$ & 6.3 & 10.0 & 2.8 \\
& $\mathrm{RE}(\%)$ & 7.2 & 0.001073 & 0.04836 \\
& Measured mean & 0.000019 & 14.0 & 4.8 \\
Day 2 & $\mathrm{hm}{ }^{5} \mathrm{C} / \mathrm{C} \%$ & 8.4 & 7.3 & -3.3 \\
$n=3$ & $\mathrm{RSD}(\%)$ & -6.9 & 0.000924 & 0.05213 \\
& $\mathrm{RE}(\%)$ & 0.000018 & 4.1 & 8.2 \\
Day 3 & $\mathrm{Measured} \mathrm{mean}$ & 5.5 & -7.6 & 4.3 \\
\hline$n=3$ & $\mathrm{hm}{ }^{5} \mathrm{C} / \mathrm{C} \%$ & -8.0 & & \\
& $\mathrm{RSD}(\%)$ & & & \\
\hline
\end{tabular}


Table S9. The accuracy and precision for the determination of $\mathrm{hm}^{2} \mathrm{G}$.

\begin{tabular}{|c|c|c|c|c|}
\hline & $\begin{array}{c}\text { Nominal } \\
\mathrm{hm}^{2} \mathrm{G} / \mathrm{G} \%\end{array}$ & 0.00020 & 0.0020 & 0.050 \\
\hline Day 1 & $\begin{array}{l}\text { Measured mean } \\
\mathrm{hm}^{2} \mathrm{G} / \mathrm{G} \%\end{array}$ & 0.00019 & 0.00208 & 0.04773 \\
\hline \multirow[t]{2}{*}{$n=3$} & RSD (\%) & 4.8 & 6.7 & 4.9 \\
\hline & RE (\%) & -6.8 & 4.1 & -4.5 \\
\hline \multirow{3}{*}{$\begin{array}{c}\text { Day } 2 \\
n=3\end{array}$} & $\begin{array}{c}\text { Measured mean } \\
\mathrm{hm}^{2} \mathrm{G} / \mathrm{G} \%\end{array}$ & 0.00021 & 0.00183 & 0.05196 \\
\hline & RSD (\%) & 2.2 & 5.4 & 1.7 \\
\hline & RE $(\%)$ & 4.3 & -8.3 & 3.9 \\
\hline \multirow{3}{*}{$\begin{array}{c}\text { Day } 3 \\
n=3\end{array}$} & $\begin{array}{c}\text { Measured mean } \\
\mathrm{hm}^{2} \mathrm{G} / \mathrm{G} \%\end{array}$ & 0.00019 & 0.00194 & 0.05093 \\
\hline & RSD (\%) & 3.7 & 3.8 & 3.8 \\
\hline & RE (\%) & -6.8 & -3.0 & 1.9 \\
\hline
\end{tabular}


Figure S1. The chemical reactions for the synthesis of (A) hm ${ }^{6} \mathrm{~A}$ and (B) $\mathrm{hm}^{2} \mathrm{G}$.

(A)<smiles>Nc1ncnc2c1ncn2C1OC(O)C(O)C1O</smiles>

Adenosine

(B)

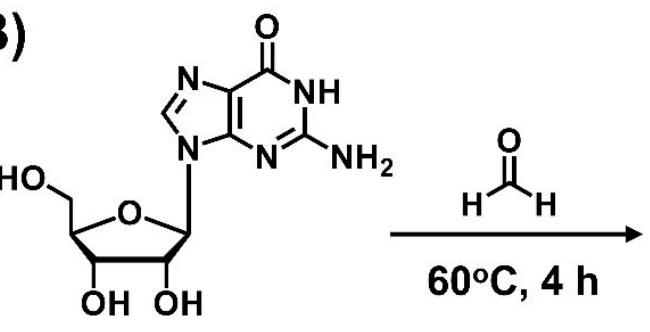

Guanosine

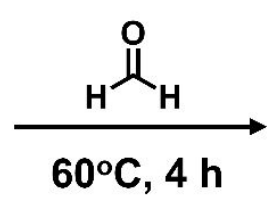<smiles>O=c1[nH]c(NCO)nc2c1ncn2C1OC2OC1C(O)C2O</smiles>
$\mathrm{hm}^{2} \mathrm{G}$ 
Figure S2. Characterization of the synthesized $\mathrm{hm}^{6} \mathrm{~A}$ and $\mathrm{hm}^{2} \mathrm{G}$ by NMR. (A) $\mathrm{hm}^{6} \mathrm{~A}$. (B) $\mathrm{hm}^{2} \mathrm{G}$.

(A)
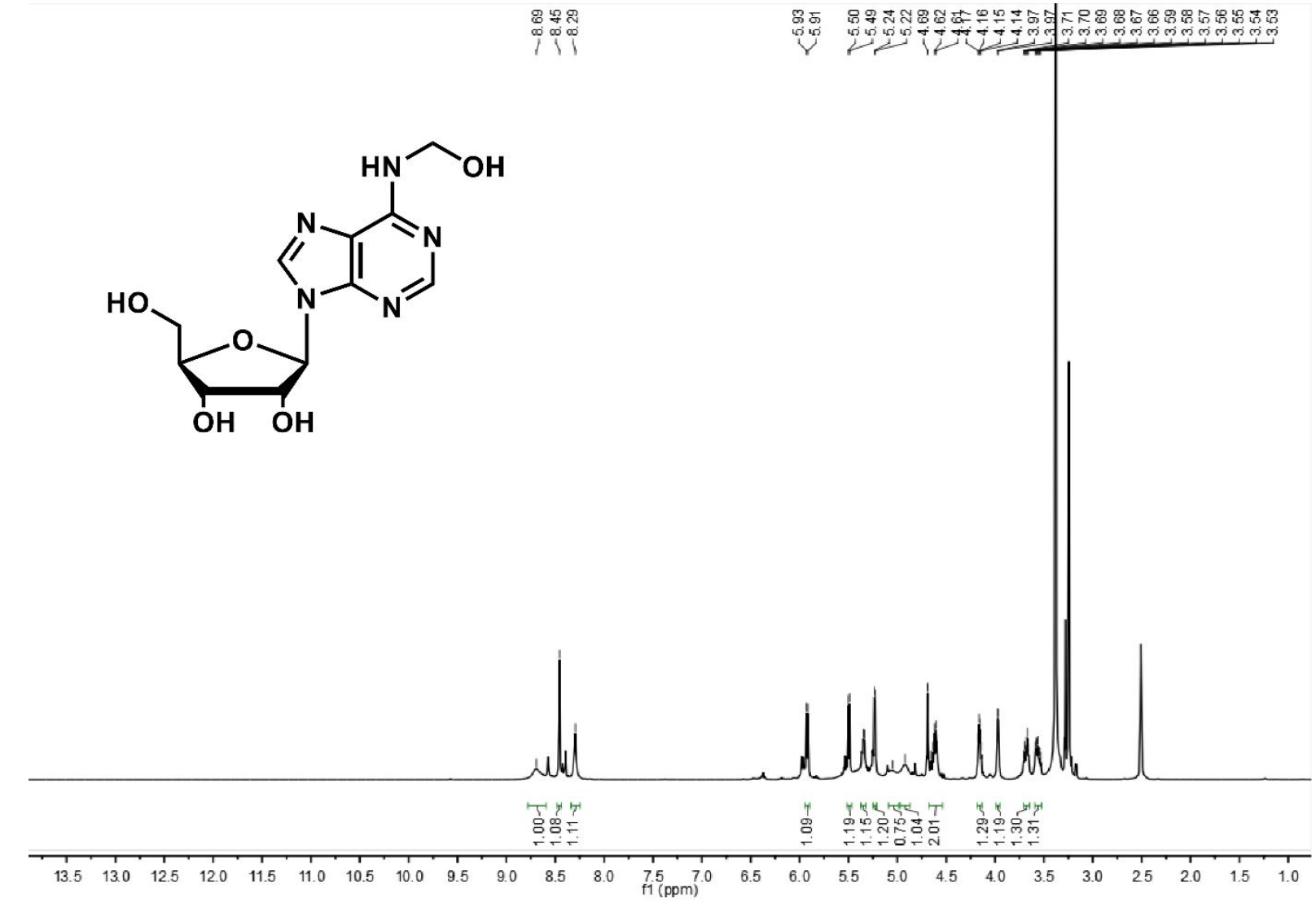

${ }^{1} \mathrm{H}$ NMR (400 MHz, DMSO- $\left.d_{6}\right) \delta 8.69(\mathrm{~s}, 1 \mathrm{H}), 8.45$ (s, 1H), $8.29(\mathrm{~s}, 1 \mathrm{H}), 5.92(\mathrm{~d}, J=6.1 \mathrm{~Hz}$, 1H), $5.50(\mathrm{~d}, J=6.2 \mathrm{~Hz}, 1 \mathrm{H}), 5.34(\mathrm{~d}, J=4.8 \mathrm{~Hz}, 1 \mathrm{H}), 5.23(\mathrm{~d}, J=4.7 \mathrm{~Hz}, 1 \mathrm{H}), 5.05(\mathrm{~s}, 1 \mathrm{H})$, $4.92(\mathrm{~s}, 1 \mathrm{H}), 4.69-4.57(\mathrm{~m}, 2 \mathrm{H}), 4.16(\mathrm{dd}, J=8.0,4.6 \mathrm{~Hz}, 1 \mathrm{H}), 3.97(\mathrm{~d}, J=3.2 \mathrm{~Hz}, 1 \mathrm{H})$, $3.68(\mathrm{dt}, J=11.8,4.1 \mathrm{~Hz}, 1 \mathrm{H}), 3.59-3.53(\mathrm{~m}, 1 \mathrm{H})$. 
(B)

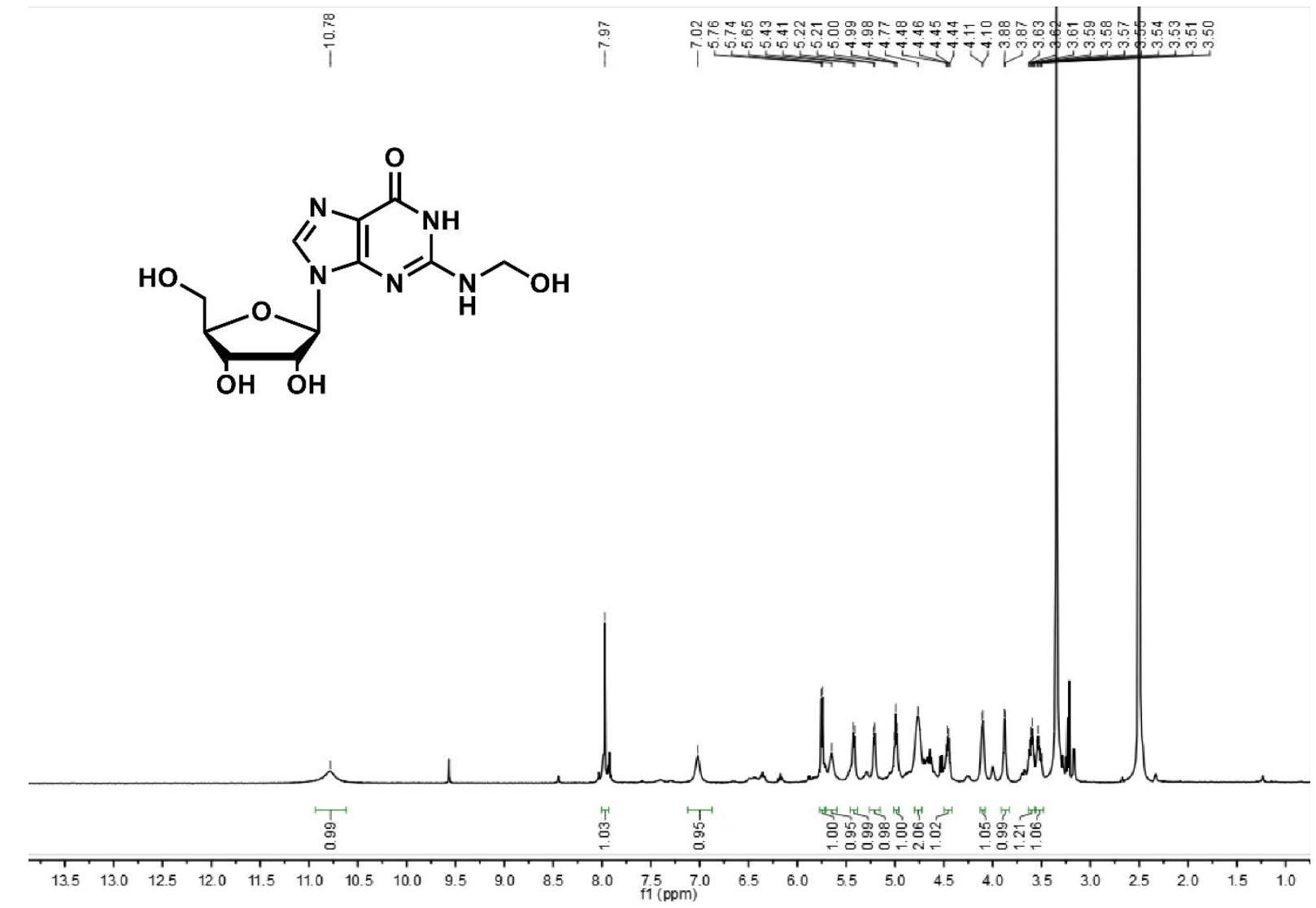

${ }^{1} \mathrm{H}$ NMR (400 MHz, DMSO-d $)_{6} \delta 10.78(\mathrm{~s}, 1 \mathrm{H}), 7.97(\mathrm{~s}, 1 \mathrm{H}), 7.02(\mathrm{~s}, 1 \mathrm{H}), 5.75$ (d, $J=6.0$ Hz, 1H), $5.65(\mathrm{~s}, 1 \mathrm{H}), 5.42(\mathrm{~d}, J=6.0 \mathrm{~Hz}, 1 \mathrm{H}), 5.21(\mathrm{~d}, J=4.7 \mathrm{~Hz}, 1 \mathrm{H}), 4.99$ (t, $J=5.4 \mathrm{~Hz}$, 1H), $4.77(\mathrm{~s}, 2 \mathrm{H}), 4.46(\mathrm{dd}, J=10.7,5.3 \mathrm{~Hz}, 1 \mathrm{H}), 4.10(\mathrm{~d}, J=4.0 \mathrm{~Hz}, 1 \mathrm{H}), 3.88(\mathrm{~d}, J=3.6$ $\mathrm{Hz}, 1 \mathrm{H}), 3.63-3.56(\mathrm{~m}, 1 \mathrm{H}), 3.56-3.46(\mathrm{~m}, 1 \mathrm{H})$. 
Figure S3. Evaluation of the stability of $\mathrm{hm}^{6} \mathrm{~A}$ and $\mathrm{hm}^{2} \mathrm{G}$ in neutral conditions $(50 \mathrm{mM}$ Tris- $\mathrm{HCl}, 10 \mathrm{mM} \mathrm{NaCl}, 1 \mathrm{mM} \mathrm{MgCl} 2,1 \mathrm{mM} \mathrm{ZnSO} 4, \mathrm{pH} 7.0$ ) at $25^{\circ} \mathrm{C}$. The decay curve is fitted into an exponential decay function.

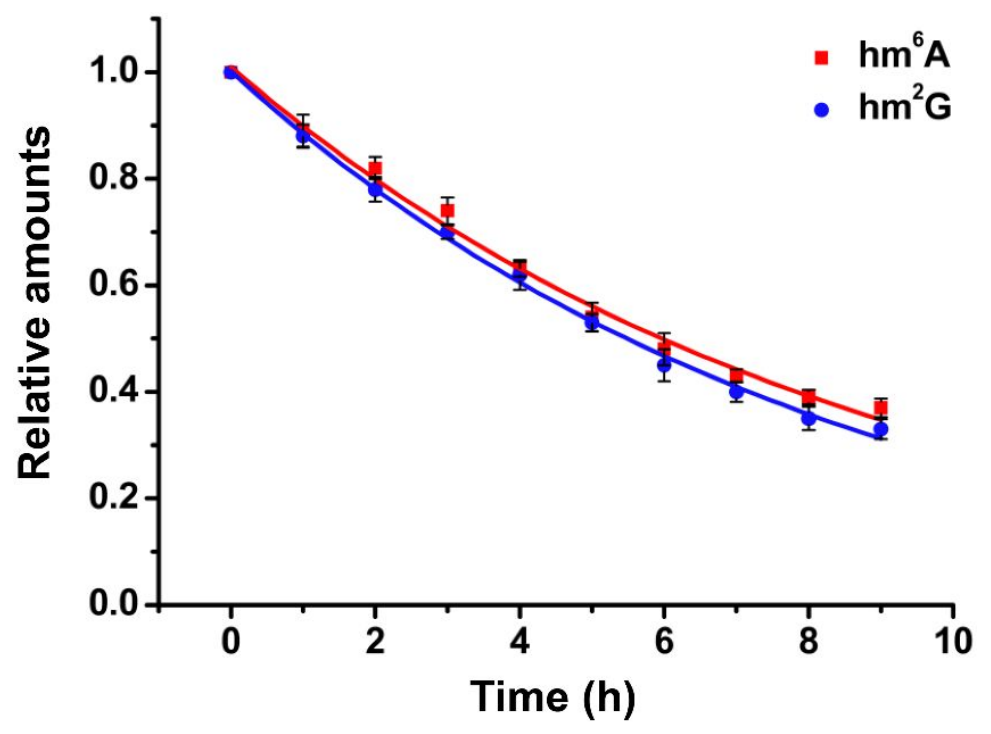


Figure S4. Evaluation of the signal intensities of normal nucleosides in neutral buffer and traditional enzymatic digestion buffer. The Y-axis shows the ratio of the signal intensities of normal nucleosides in neutral buffer over in traditional enzymatic digestion buffer.

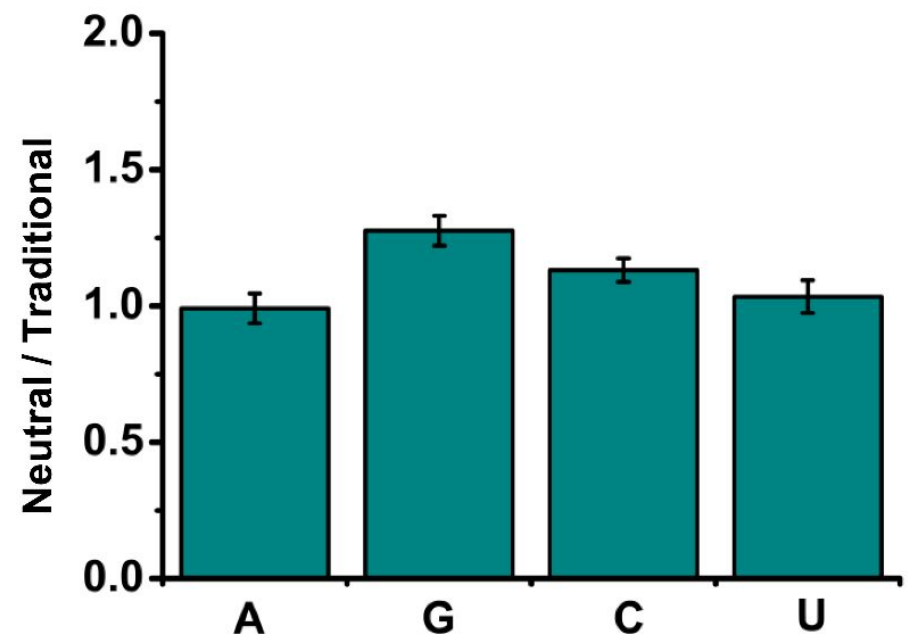


FigureS5. Confirmation of the detected $\mathrm{hm}^{6} \mathrm{~A}, \mathrm{hm}^{5} \mathrm{C}$, and $\mathrm{hm}^{2} \mathrm{G}$ by spiking the standards to the tested samples. (A) The extracted-ion chromatograms of the $\mathrm{hm}^{6} \mathrm{~A}$ standard (upper panel), detected $\mathrm{hm}^{6} \mathrm{~A}$ from RNA of HEK293T cells (middle panel), and the detected $\mathrm{hm}^{6} \mathrm{~A}$ from RNA of HEK293T cells with spiked $\mathrm{hm}^{6} \mathrm{~A}$ standard (bottom panel). (B) The extracted-ion chromatograms of the $\mathrm{hm}^{5} \mathrm{C}$ standard (upper panel), detected $\mathrm{hm}^{5} \mathrm{C}$ from RNA of HEK293T cells (middle panel), and the detected $\mathrm{hm}^{5} \mathrm{C}$ from RNA of HEK293T cells with spiked $\mathrm{hm}^{5} \mathrm{C}$ standard (bottom panel). (C) The extracted-ion chromatograms of the $\mathrm{hm}^{2} \mathrm{G}$ standard (upper panel), detected $\mathrm{hm}^{2} \mathrm{G}$ from RNA of HEK293T cells (middle panel), and the detected $\mathrm{hm}^{2} \mathrm{G}$ from RNA of HEK293T cells with spiked $\mathrm{hm}^{2} \mathrm{G}$ standard (bottom panel).

(A)

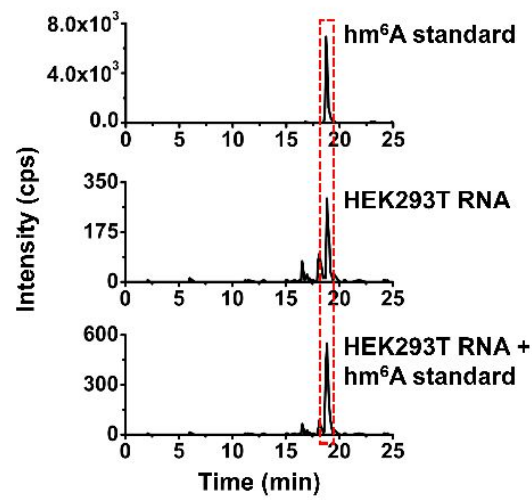

(B)

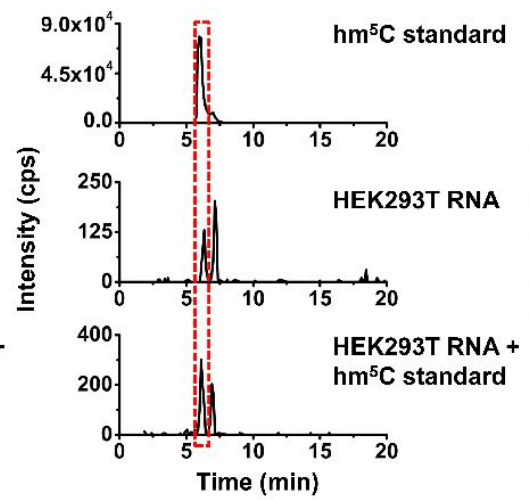

(C)

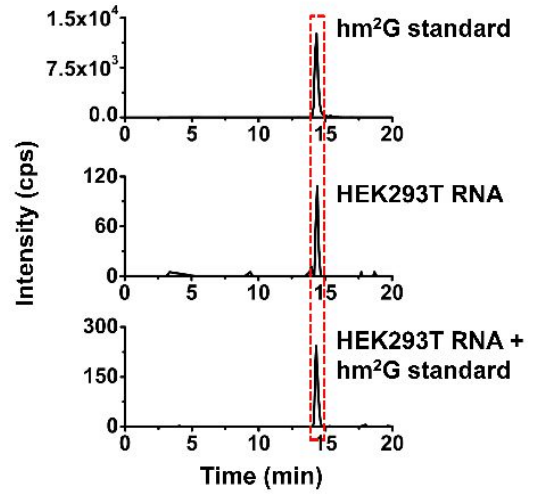


Figure S6. The extracted-ion chromatograms of the samples with only adding phosphodiesterase I or calf intestinal alkaline phosphatase (CIAP) and omitting the RNA sample.

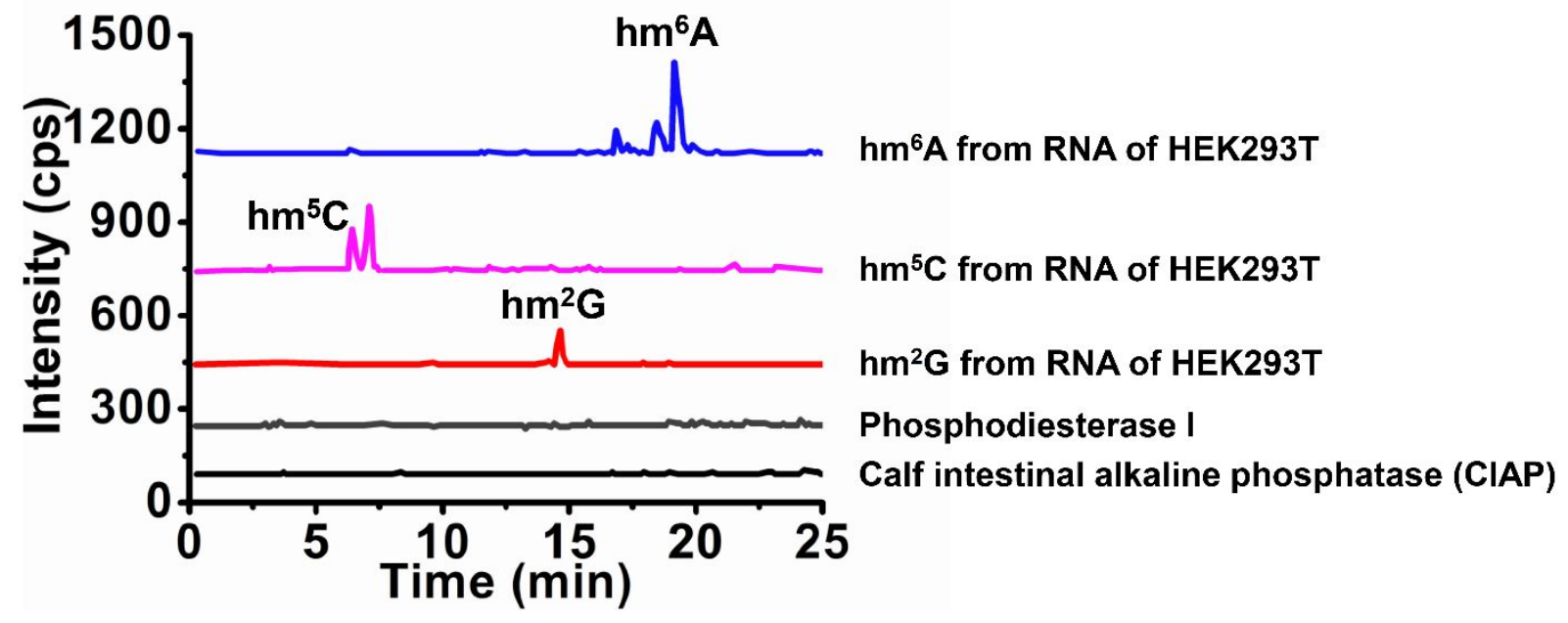


Figure S7. The typical extracted-ion chromatograms and product ion spectra of $\mathrm{m}^{6} \mathrm{~A}, \mathrm{~m}^{5} \mathrm{C}$, and $\mathrm{m}^{2} \mathrm{G}$ from standards and from RNA of HEK293T cells.

(A)
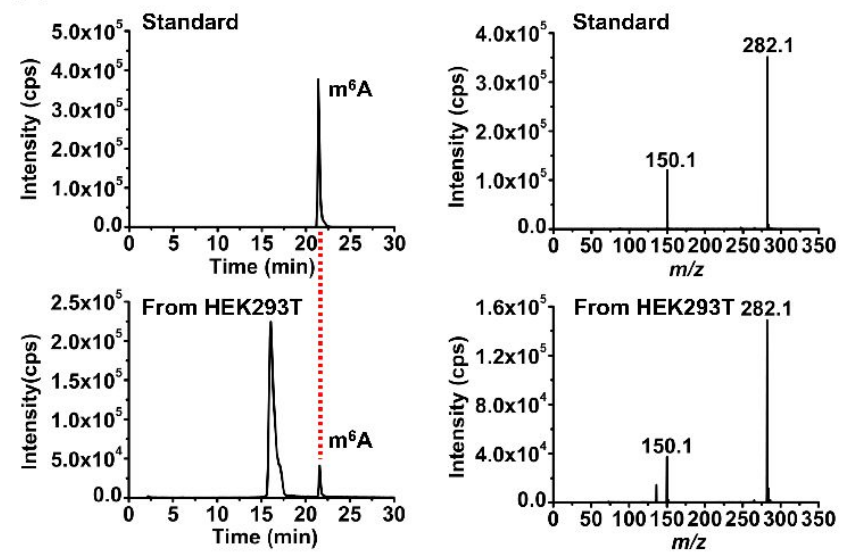

(B)
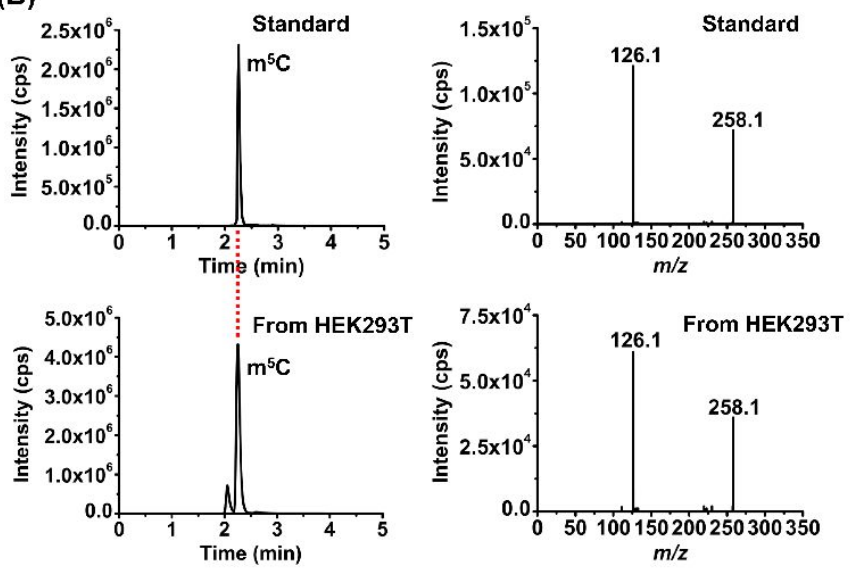

(C)
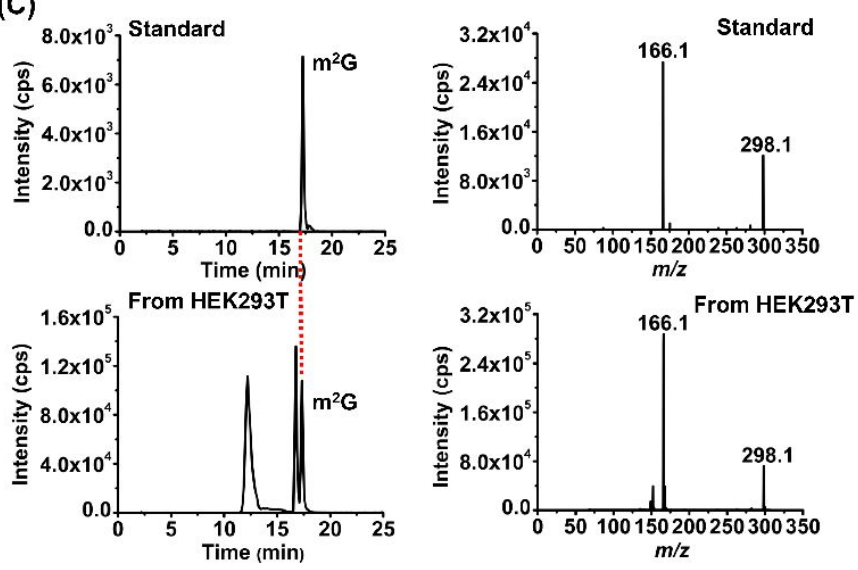
Figure S8. Confirmation of the detected $\mathrm{m}^{6} \mathrm{~A}, \mathrm{~m}^{5} \mathrm{C}$, and $\mathrm{m}^{2} \mathrm{G}$ by spiking the standards to tested samples. (A) The extracted-ion chromatograms of the $\mathrm{m}^{6} \mathrm{~A}$ standard (upper panel), detected $\mathrm{m}^{6} \mathrm{~A}$ from RNA of HEK293T cells (middle panel), and the detected $\mathrm{m}^{6} \mathrm{~A}$ from RNA of HEK293T cells with spiked $\mathrm{m}^{6} \mathrm{~A}$ standard (bottom panel). (B) The extracted-ion chromatograms of the $\mathrm{m}^{5} \mathrm{C}$ standard (upper panel), detected $\mathrm{m}^{5} \mathrm{C}$ from RNA of HEK293T cells (middle panel), and the detected $\mathrm{m}^{5} \mathrm{C}$ from RNA of HEK293T cells with spiked $\mathrm{m}^{5} \mathrm{C}$ standard (bottom panel). (C) The extracted-ion chromatograms of the $\mathrm{m}^{2} \mathrm{G}$ standard (upper panel), detected $\mathrm{m}^{2} \mathrm{G}$ from RNA of HEK293T cells (middle panel), and the detected $\mathrm{m}^{2} \mathrm{G}$ from RNA of HEK293T cells with spiked $\mathrm{m}^{2} \mathrm{G}$ standard (bottom panel).

(A)

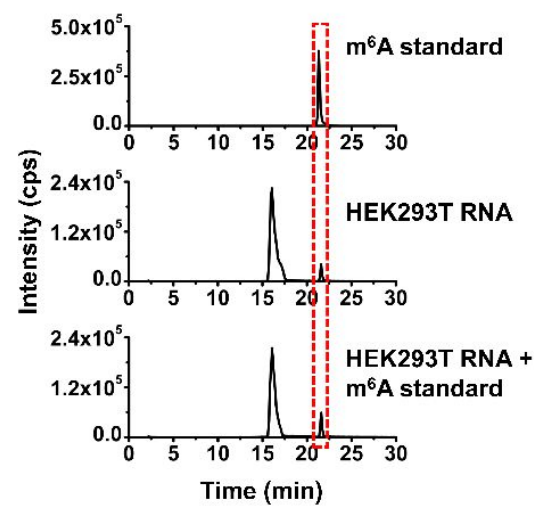

(B)

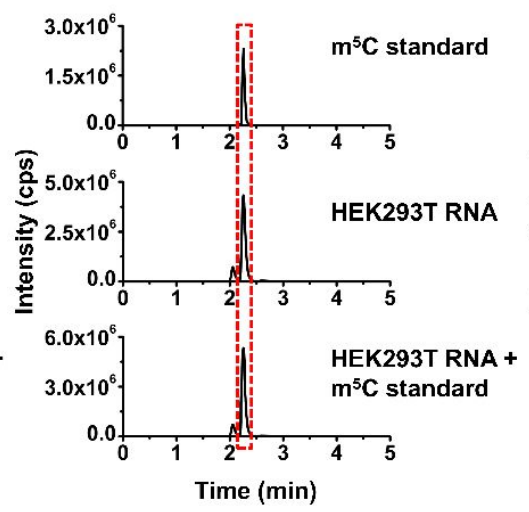

(C)

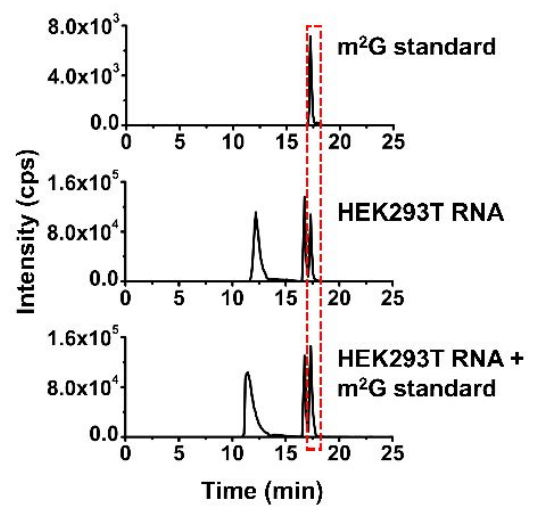

